\title{
How many patients can each surgeon have on their waiting list and still treat them all in time?
}

\author{
Martin J. Connor, Danielle Ben Bashat, Christopher D. Ogg \\ Centre for Applied Health Economics, Menzies Health Institute, Griffith University, Australia
}

Received: September 11, 2020

Accepted: September 15, 2020 Online Published: September 22, 2020

DOI: $10.5430 /$ jha.v9n4p39

URL: https://doi.org/10.5430/jha.v9n4p39

\begin{abstract}
Objective: In many advanced economies patients wait on elective surgery waiting lists longer than clinically recommended times. This results from either a demand and capacity differential or challenges with the chronological management of patient bookings. This paper describes a novel algorithm that calculates elective surgery capacity and demand imbalances at a surgeon and urgency category level.

Methods: An algorithm was developed that is surgeon-specific, sensitive to clinical urgency, relates to patient- and procedure level, and is scalable, dynamic and efficient. The novel measure designated the "Nominal Waiting List Maximum", uses historic waiting list removal rates to approximate waiting list capacity at a surgeon- and urgency category-level. This measure can then be compared to the actual patients on each surgeon's waiting list for each urgency category at a given point in time to measure imbalances.

Results: In 2014, the algorithm was automated and implemented across a large Hospital and Health Service (HHS), in QLD, Australia, within an analytics solution. The solution extracts current and historic elective surgery waiting list episode-level data from underlying repositories and calculates "Nominal Waiting List Maximum" for every surgeon at an urgency category level with daily data flows.

Conclusions: The solution helped the large tertiary hospital group to identify demand and capacity imbalances at a surgeon and urgency category level to improve theatre session allocations. With the aid of this measure, the HHS achieved zero patients waiting longer than clinically recommended times and was able to hold this position for more than 2 years demonstrating the value of this algorithm. The solution was subsequently rolled out to 55 hospitals across QLD, Australia and anonymised views provided to the hospitals' governing body.
\end{abstract}

Key Words: Hospital management, Capacity and demand, Elective surgery, Surgeons, Waiting list, Waiting times

\section{INTRODUCTION}

Patients waiting longer than clinically recommended timeframes for elective surgery and other planned care services is a worldwide challenge and an important policy issue. ${ }^{[1-4]}$ Long delays can enable symptoms to deteriorate, and frustrate patients, surgeons and supporting staff. ${ }^{[5,6]}$ The effi- ciency of the scheduled care system is immensely important for hospital stakeholders as surgery is often the greatest cost and source of revenue for hospitals, at times accounting for up to $60 \%-70 \%$ of hospital admissions. ${ }^{[7]}$

Extended waiting times for elective surgery can be caused by a mismatch between waiting list demand and capacity, result-

\footnotetext{
*Correspondence: Martin J. Connor; Email: martin.connor@ healthcarelogic.com; Address: Healthcare Logic, Level 4, 64 Marine Parade, Southport, QLD, 4215, Australia.
} 
ing from inefficient capacity planning, ${ }^{[8]}$ or challenges with the chronological management of patient bookings (process issues). In recent years, the demand for elective surgery has increased, ${ }^{[9]}$ which is likely linked to aging populations, the introduction of new technologies and patients' expectations for better health outcomes. ${ }^{[10]}$ Simultaneously, healthcare policy makers and stakeholders struggle to match capacity to the specific surgery demand, which varies based on outpatient throughput and patient cohorts.

Policies that aim to reduce the number of patients waiting more than clinically recommended times (long wait patients) often attempt to reduce the demand or increase the capacity by funding extra theatre sessions or by outsourcing patients. ${ }^{[11]}$ However, reducing demand even when taking into consideration clinical and non-clinical parameters, may raise ethical dilemmas ${ }^{[11]}$ and various studies concluded that demand may not necessarily be the cause for long waiting times. ${ }^{[5]}$ Interestingly, other studies have suggested that the lack of capacity is not the major issue and funding extra sessions may be a suboptimal approach from a financial perspective, ${ }^{[8]}$ but rather the root cause of patients waiting longer than clinically recommended timeframes is a combination of demand and capacity variation in conjunction with ineffective capacity planning. ${ }^{[8]}$ Hence, there is a need for a targeted demand and capacity monitor to enable risk escalations and more effective theatre allocations. ${ }^{[12]}$

An effective analysis of demand and capacity must consider the individual surgeon's capacity; their ability to treat or remove patients from the waiting list. Waiting list removal rates differ between surgeons and may be impacted by a surgeon's specialty, age, experience, teaching sessions, as well as personal circumstances and other hospital commitments. ${ }^{[13,14]}$ Thus, we must bear in mind that a surgeon's capacity is specific to each individual and is dynamic, and so its evaluation should be updated frequently.

Waiting lists provide flexibilities for patients to choose the optimal treatment date available and for hospitals to manage the variation in demand with other factors (theatre session availability, inpatient bed availability, staff leave etc.). Government policies specify accepted criteria to categorize patients into urgency categories with maximum waiting times, aiming to preserve patient equity and provide a measurable quality framework. Despite these efforts and the importance of the scheduled care pathway, few published studies have focused on methods to calculate maximum or manageable waiting lists sizes, which serve as leading indicators of long wait patients. Furthermore, studies that do are not surgeonor category-specific nor dynamic, ${ }^{[15]}$ which can limit the practical implementation of such models.
At present, there is a lack of a standard definition and methodology to calculate maximal waiting list sizes. In this study, we address this issue by proposing a clear definition and describing the algorithm we developed to determine the nominal maximal waiting list sizes for each surgeon at an urgency category level. This algorithm was embedded in an automated decision-support tool that allows an easy identification of waiting lists with a demand and capacity imbalance; to identify both "heavy" and "light" waiting lists for more effective capacity planning. In this paper, we demonstrate the usability of our algorithm and summarize the results when implementing across a large Hospital and Health Service (HHS), in QLD, Australia.

\section{METHODS}

\subsection{Properties of the algorithm}

In the absence of a published answer to our question, we designed our algorithm with the following characteristics.

1. Sensitive to clinical urgency - we know that clinical priority is the cornerstone of resource allocation for safe and ethical clinical practice, and that any capacity and demand algorithm that did not fundamentally rest on clinical priority will be prima facie unacceptable professionally.

2. Surgeon-specific - we know that each surgeon's practice is importantly unique. From case mix, to the range of morbidity, to the technology and team supports available, to differences related to style and clinical preferences, there are a range of variables meaning that measures that apply even at specialty or sub-specialty level will not be sufficiently sensitive. This is particularly important since we want to be able to use the product of the algorithm to identify capacity and demand risk, which is best expressed at individual surgeon level.

3. Patient-and procedure-level - since we want to pinpoint capacity and demand issues in a way that is actionable, and we are mostly aiming to support surgeons and teams in public hospitals (though our logic is equivalently applicable to private settings), it is important for our algorithm to run over patient and procedure level information. This enables surgeons and their support teams to be well informed when designing changes to improve demand and capacity balance, whether this is moving patients around within a team, arranging for increased operating capacity, accessing private sector capacity, reducing the number of patients flowing on to the surgical waiting list, or some other tactical approach.

4. Scalable - whilst we want to be able to calculate the ideal waiting list size for each surgeon, it is also important for us to be able to run the algorithm across all surgeons in a practice or hospital. This is essential because before we run 
the calculation, there is by definition no quantifiable means of establishing where capacity and demand imbalances are occurring within, or between, teams and specialties.

5. Dynamic - we know that surgical practice is not static. Allocations of theatre time, changes in practice, variations in listing rates linked to the volume or morbidity in outpatient clinics and changes in case mix occur at different moments for different surgeons. We want to be able to understand how each of these changes feeds through and ideally quantify the impact on the future ability of each surgeon to maintain delivery of a maximum waiting time in the context of the overall balance of their practice.

6. Efficient - there is limited value in developing an algorithm that would have very high implementation costs or would require a major exercise of case-note reviews before being capable of supporting an analysis of capacity and demand. This requirement led us to design an approach that could be implemented using only the information that is routinely available in public hospitals and would typically not require any significant new data collection. This pursuit of efficiency also led us to develop software to automate the extraction of the relevant data from hospital systems on a daily basis without the requirement of manual intervention.

\subsection{The algorithm and its variables}

Our algorithm starts by running counts for each surgeon of every patient in each clinical urgency category that has been treated in the last 12 months. This gives us the "treatment rate" for each surgeon and personalises the results to their practice. Since we look at treatment rates by clinical urgency category, we ensure that results reflect this as a basic property.

The algorithm then runs counts for all patients removed for reasons other than treatment. These are patients who have been added to the surgeon's waiting list at a point in time but are then taken off the list without being treated. There are numerous reasons why this can happen, from patients changing their mind, being treated elsewhere, moving to a different area or being too ill for the proposed treatment. Incorporating removals for reasons other than treatment is essential, since variation in this number has a significant impact on the amount of capacity required to treat an overall waiting list at each point in time.

The algorithm then sums these counts together to give us a combined "propensity to remove" rate for each surgeon and for each urgency category. This annual removal rate is then divided by 12 to give a mean monthly removal rate for each urgency category.

The algorithm then uses the maximum treatment times that are to be used for each urgency category. These maximums are typically set at government level for public hospitals, but they can be set at hospital level. In Australia, where we first developed this technique there are three standard categories for urgency each with their own maximum waiting time: category one cases should be seen within 30 days, category two cases within 90 days and category three cases within 365 days.

We can then take the mean monthly removal rates for each surgeon, and multiply them by the number of months permitted by the maximum treatment time. In Australia, as described above, this is one month for category one patients, three months for category two patients and twelve months for category three patients. This yields what we call the "nominal waiting list maximum", personalised for each surgeon in each urgency category. (We use the label of "nominal" to capture the fact that it is a useful pragmatic estimate of a surgeon's capacity, accepting that it depends for its value on the assumption that the near future will be like the recent past in terms of treatment and removal rates).

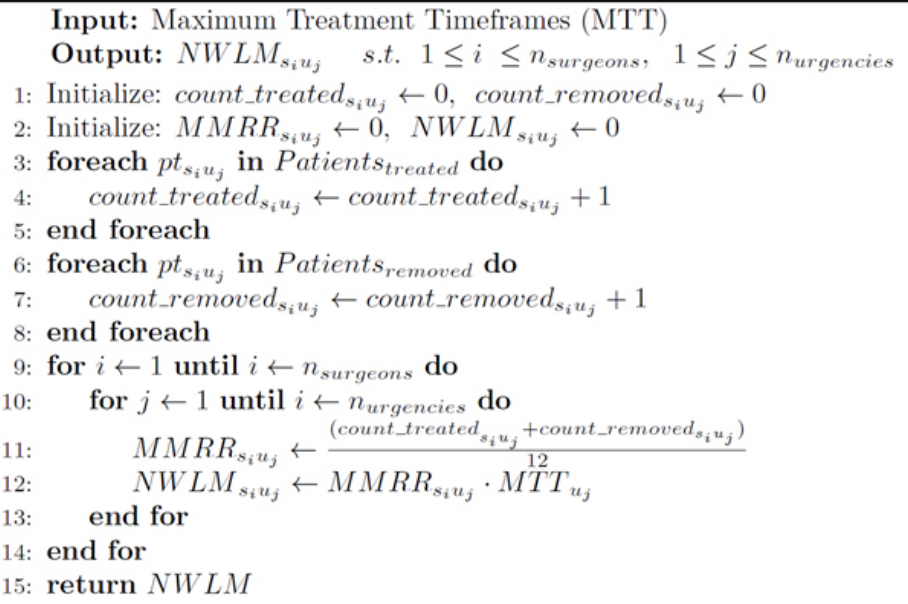

Published by Sciedu Press
Figure 1. The algorithm for calculating 'Nominal Waiting List Maximums' at a surgeon and category level 
Figure 2. The algorithm for determining a demand-capacity imbalance at a surgeon and category level

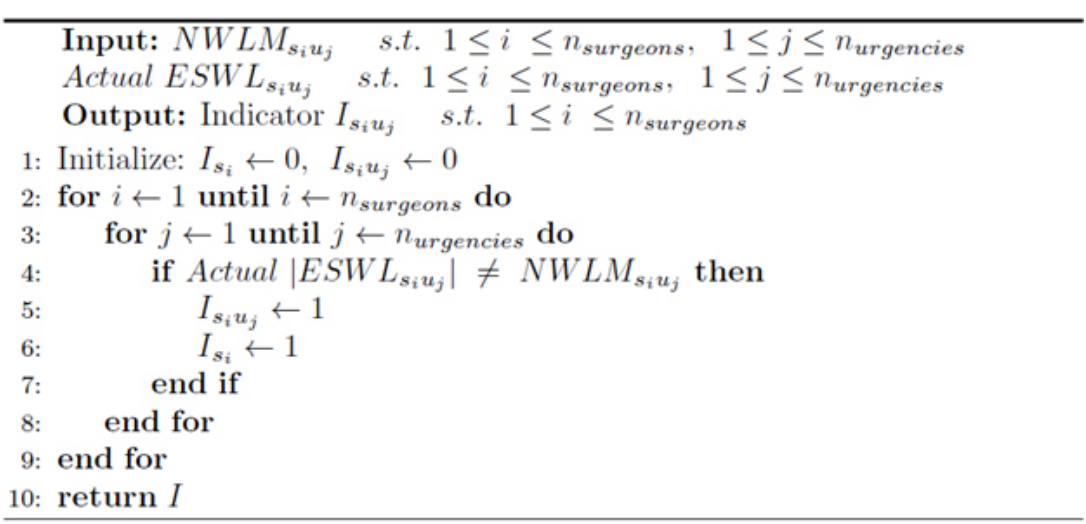

So for example, if a surgeon has a mean monthly removal rate in the last year of five category one patients, ten category two patients and twenty category three patients, then their "nominal waiting list maximums" will be five patients for their category one list, thirty patients for their category two list and 240 patients for their category three list (see Figure 1).

The algorithm completes by comparing the "nominal waiting list maximum" numbers for each surgeon and urgency level by the actual waiting list each morning (which includes all the additions and removals from the previous day) to provide an indicator of implied capacity and demand imbalance wherever the actual waiting list sits above the nominal waiting list maximum (see Figure 2).

\subsection{Practical application}

The application of this algorithm gains significant utility when it is incorporated into software that can automate the entire process - from the extraction and maintenance of the "trailing" twelve month averaging calculations, to the monitoring of the daily list by surgeon and urgency category. The same software - that we have developed using co-design processes with a wide variety of surgical teams - also visualises trends of both individual surgeon's actual waiting lists, and our calculations of the respective nominal waiting list maximums. When developed into charts that can also identify any surgeons with patients waiting beyond the maximums allowed, we then have a quantified approach to capacity and demand analysis that can operate automatically and meet all the design requirements listed above.

A sample of what the output of the algorithm looks like can be seen in Table 1 that shows each contributing variable and calculations across the three urgency categories in Australia. This output enables each surgeon to see their own calculation, and this transparency facilitates both establishing "face validity" for the counts (to ensure the activity data and surgeon names are mapped correctly) and shows the calculated nominal waiting list maximums for each urgency category.

In the example shown in Table 1, we can see the signal is true for a capacity and demand imbalance, and also that the problem is occurring with the category two cohort.

Table 1. Output of the algorithm

\begin{tabular}{lccc}
\hline Doctor & DoctorCode-7138 & DoctorCode-7138 & 2 \\
\hline Category & 1 & 3 & 12 \\
Months Active & 1 & 3 & 12 \\
Max. Treatment Timeframe (Months) & 28 & 128 \\
Patients Treated In Previous 12 Months & 1 & 15 \\
Patients Removed Other Than Treatment From ESWL. In Previous 12 Months & 29 & 143 \\
Total Patients Treated Or Removed From the ESWL In Last 12 Months & 3 & 11 \\
Average Patients Removed From The ESWL Per Month & 3.6 & 104 \\
Nominal List Max. By Max. Treatment Timeframe & 1 & 35.8 \\
Actual ESWL As At Today & $27.60 \%$ & 104 \\
Present ESWL As A \% Of Nominal Maximum Capacity & $124.10 \%$ & $209.80 \%$ \\
Category 1-3 Summary By Doctor & $98.10 \%$ & $124.10 \%$ \\
\hline
\end{tabular}


Once we have established the nominal waiting list maximum - and automated its calculation - we can then trend this number itself. To further explore the case above, we could look at the most recent 12-month trend of our example surgeon's category two nominal maximum as shown in Figure 3.

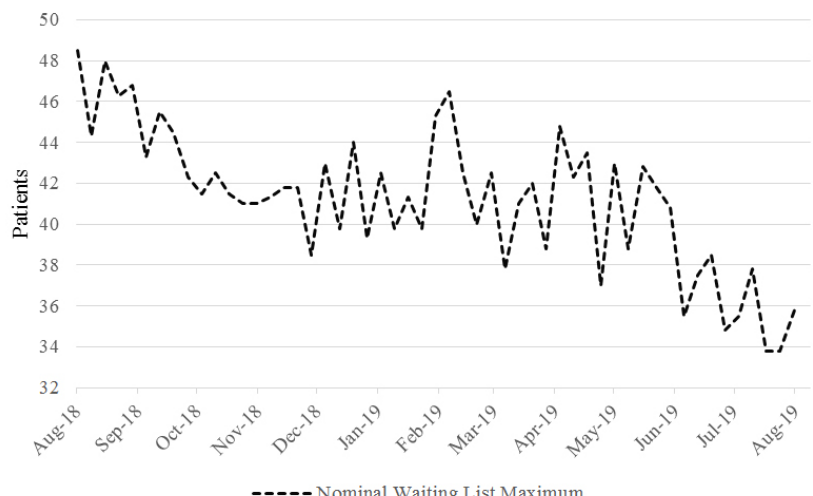

Figure 3. The trend of a surgeon's category 2 nominal waiting list maximum showing a decrease over time

As shown in Figure 3, the declining value of the nominal maximum over time demonstrates its sensitivity to the actual changes in treatment and removal rates. This graph shows a situation where this surgeon has in fact treated and/or removed fewer patients within urgency category 2 , which is reflected by our measure and demonstrates that the "order book" the surgeon can carry and still treat all their patients in time has to reduce in a linear relationship to their treatment and removal rate.

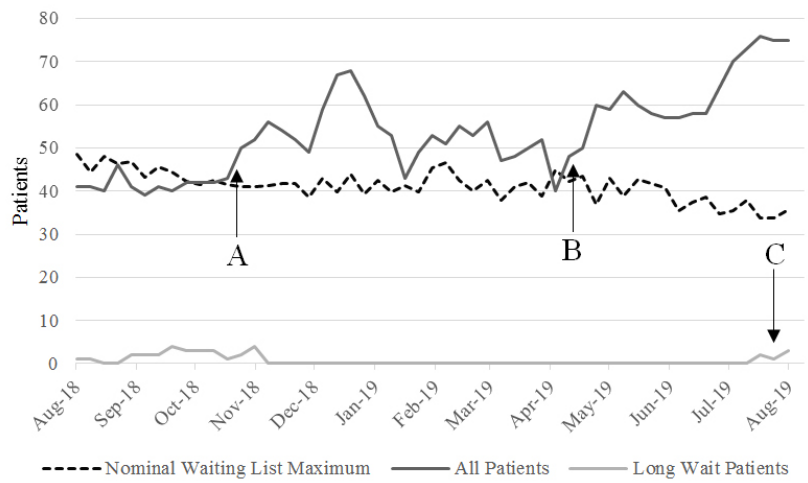

Figure 4. The trend of a surgeon's category 2 nominal waiting list maximum, all patients waiting and the long wait patients

For completeness, if we then overlay the trend lines of the same surgeon's daily actual waiting list, and a trend line showing daily counts of patients who have been waiting beyond the maximum waiting time (in this example, 90 days), we get a powerful summary of the capacity and demand situation for this surgeon and this urgency category, as can be seen in Figure 4.

Figure 4 brings all the outputs of the algorithm together and highlights a situation that occurred at Point A where the waiting list increased beyond that of the nominal waiting list maximum. The Team was alerted of this position and were able to bring the waiting list back into balance. However, this was not the case at Point B which interestingly shows the correlation between the divergence of the daily actual waiting list and the calculated nominal maximum with the long waits position in August (Point C). We can see the predictive force apply as the number of long waiting patients begins to appear 90 days after the divergence.

For completeness, we provide an additional example of a nominal waiting list maximum, which rises over time demonstrating its sensitivity to increasing treatment and removal rates, shown in Figure 5.

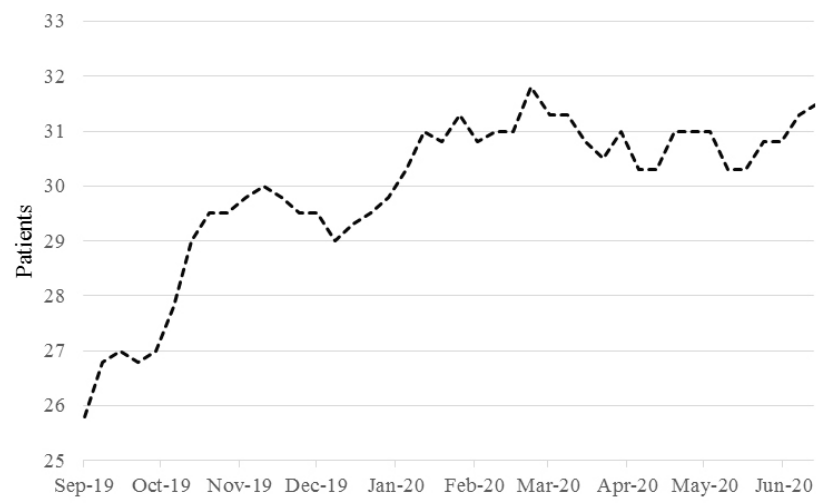

Figure 5. The trend of a surgeon's category 2 nominal waiting list maximum showing an increase over time

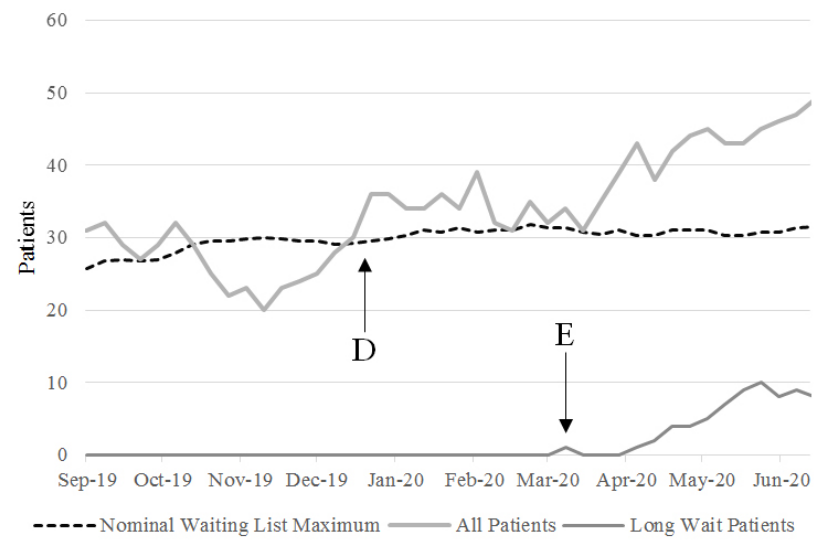

Figure 6. Capacity and demand status per surgeon and category level 2 
Showing again a summary of the capacity and demand situation for surgeon and the urgency category (see Figure 6), we can see the waiting list increase above the nominal waiting list maximum (Point $\mathrm{D}$ ) which is correlated with long wait patients three months later (from Point E).

\section{RESUlts}

Using the software that incorporated the nominal waiting list maximums, clinical and managerial teams within the HHS were able to identify surgeons with current waiting list positions above their nominal waiting list maximums and together formulate strategies to bring these lists into balance. These strategies included funding targeted short-term additional theatre sessions to bring lists into balance, and minor increases of the theatre template for specific surgeons to help maintain balance going forward. Reviews of patients on "heavy" order books were performed to confirm the appropriateness of the patient categorizations, to audit the data for errors and to determine if it was appropriate for certain patients to be treated by other team members (with lighter order books); the patient-level information was accessible within the software.

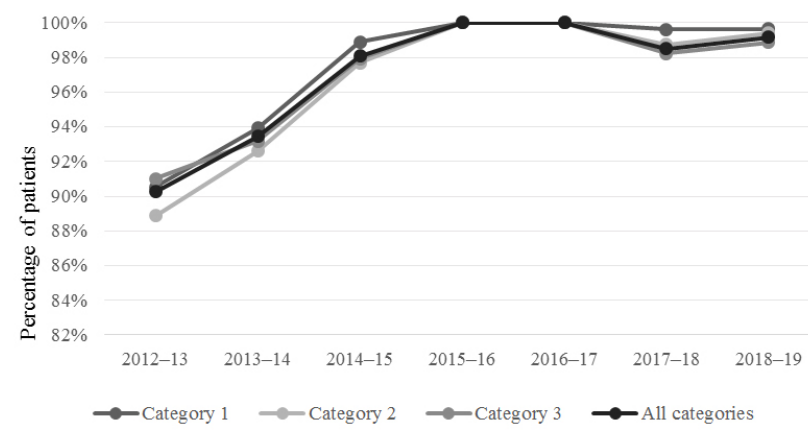

Figure 7. Elective surgery percentage of patients treated within the clinically recommended time by category

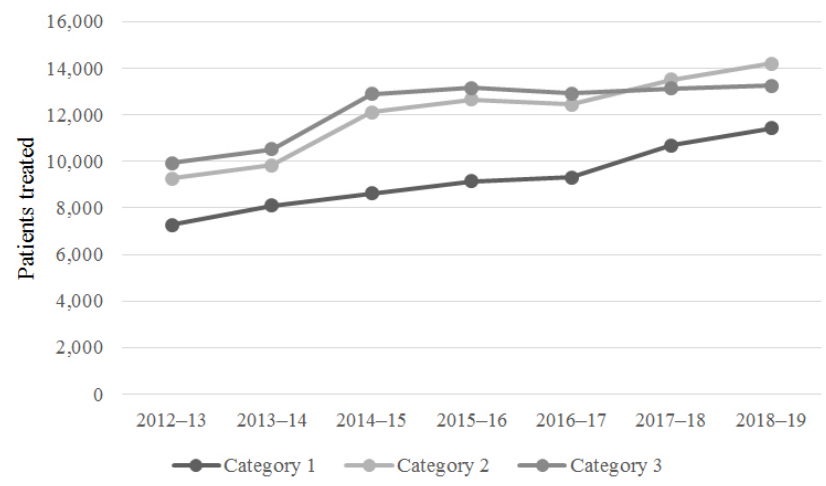

Figure 8. Elective surgery number of total patients treated within the clinically recommended time by category
For demonstrating the value of this algorithm, a year by year data was retrieved from Australian Institute Health and Welfare (AIHW) website. ${ }^{[16]}$ The HHS implemented the solution in late 2014 and was able to achieve zero patients waiting longer than clinically recommended times and hold this position for several years as can be shown in Figure 7. The volume of elective cases treated at the HHS per financial year are shown for completeness in Figure 8.

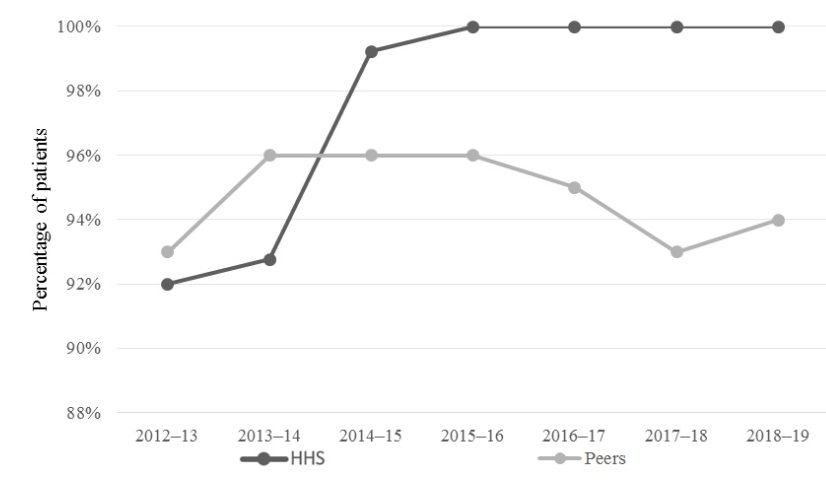

Figure 9. The percentage of category 1 elective surgery patients treated within clinical recommended time for the HHS that implemented the nominal waiting list maximum solution and its Australian peer group hospitals

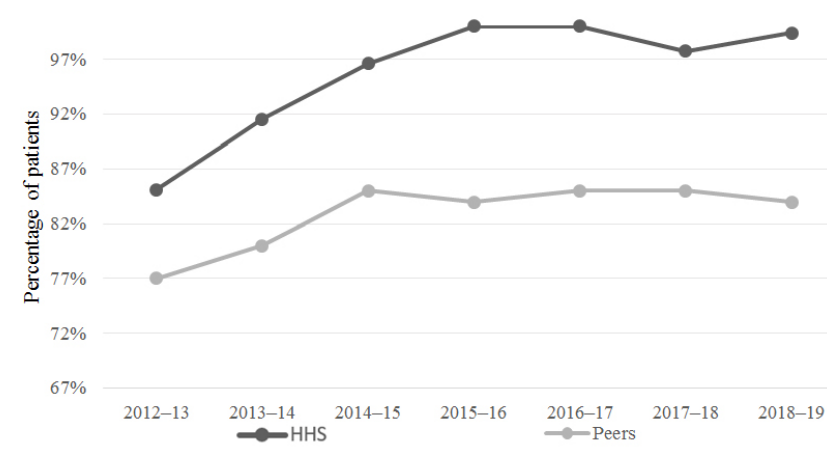

Figure 10. The percentage of category 2 elective surgery patients treated within clinical recommended time for the HHS that implemented the nominal waiting list maximum solution and its Australian peer group hospitals

\section{Discussion}

The novel algorithm described in the paper, the nominal waiting list maximum, when combined with the current waiting list positions was used to determine demand and capacity imbalances at a surgeon and category level. It serves as a leading indicator of future long wait patients and also identifies Surgeons with "light order books". For the latter cohort, this measure can provide assurance that an increase in waiting list sizes (up to the nominal waiting list maximum) will not result in long wait patients and so can support discussions of increasing outpatient throughput or alternatively reallocating 
theatre session. It was interesting that, prior to the existence of this algorithm, a large number of Surgeons managed their waiting lists close to their nominal waiting list maximums this may be a topic for a follow up study.

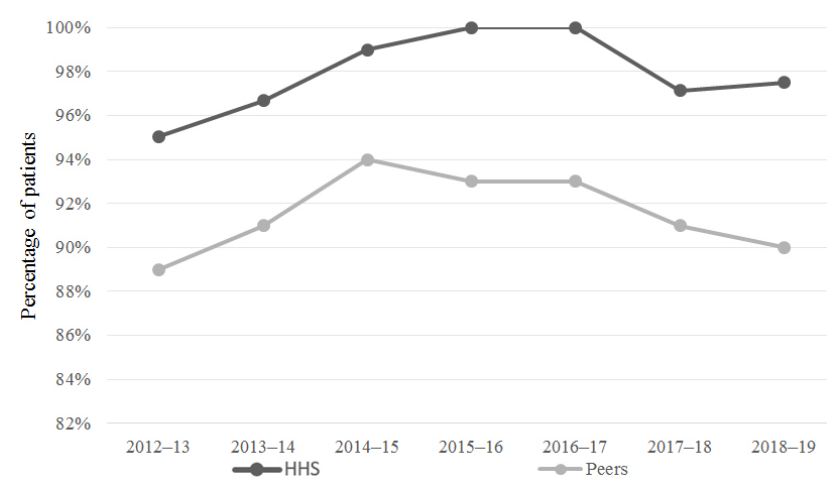

Figure 11. The percentage of category 3 elective surgery patients treated within clinical recommended time for the HHS that implemented the nominal waiting list maximum solution and its Australian peer group hospitals

The algorithm was implemented within an automated software at a large tertiary HHS in QLD. Following the implementation of the automated solution that includes the nominal waiting list maximum calculations, the HHS increased its performance relative to its Australian peer group hospitals for category 1 and category 2 patients, and maintained its high performance for category 3 patients, as can be seen in Figures 9-11. The figures were created by data obtained from AIHW website. ${ }^{[16]}$

The algorithm was designed to be surgeon- and urgencycategory specific, which aided discussions between clinicians and hospital management teams. The solution's uptake with clinical teams was also enhanced by the algorithm's relative simplicity, and the solution's ability to show each Surgeon their current waiting list by procedure and at a patient level.

Following the success of the solution's implementation as demonstrated by the HHS, the software containing the algorithm was rolled out to 55 hospitals across QLD demonstrating the efficiency and scalability of the algorithm.

\section{Conclusions}

This paper describes a novel, dynamic, scalable algorithm that can be used to determine the nominal maximal waiting list sizes for each surgeon at an urgency category level. The algorithm enables the identification of waiting lists that are "heavy", "light" or in balance with respect to waiting list demand and each surgeon's propensity to remove patients from their list.

\section{CONFLicts OF INTEREST Disclosure}

The authors now work for Healthcare Logic Pty Ltd, a public interest company that produces commercial software for capacity and demand analyses in Public Hospitals.

\section{REFERENCES}

[1] Canadian Institute for Health Information. Wait times longer for joint replacements and cataract surgeries in Canada. 2018 [cited 202024 April]. Available from: https ://www.cihi.ca/en/wait-times-longer-for-j oint-replacements-and-cataract-surgeries-in-canada

[2] Australian Institute of Health and Welfare. More patients, longer waiting times for both elective surgery and emergency department care. 2019 [cited 202024 April]. Available from: https: //www . ai hw.gov.au/news-media/media-releases/2019/december/ more-patients-longer-waiting-times-for-both-electi

[3] OECD. Waiting times for elective surgery. 2018.

[4] OECD. Waiting times for elective surgery. 2019.

[5] Lungu DA, Ruggieri TG, Nuti S. Decision making tools for managing waiting times and treatment rates in elective surgery. BMC Health Services Research. 2019; 19(1): 369. PMid: 31185989. https ://doi.org/10.1186/s12913-019-4199-6

[6] Mache S, Vitzthum K, Klapp BF, et al. Improving quality of medical treatment and care: are surgeons' working conditions and job satisfaction associated to patient satisfaction? Langenbeck's Archives of Surgery. 2012; 397(6): 973-982. PMid: 22638703. https://doi.org/10.1007/s00423-012-0963-3

Published by Sciedu Press
[7] Guerriero F, Guido R. Operational research in the management of the operating theatre: a survey. Health Care Management Science. 2011; 14(1): 89-114. PMid: 21103939. https://doi.org/10.1007/s1 0729-010-9143-6

[8] Silvester K, Lendon R, Bevan H, et al. Reducing waiting times in the NHS: is lack of capacity the problem? Clinician in Management. 2004; 12(3).

[9] Weiser TG, Haynes AB, Molina G, et al. Size and distribution of the global volume of surgery in 2012. Bull World Health Organ. 2016; 94(3): 201-209F. PMid: 26966331. https ://doi.org/10.2471/ BLT. 15. 159293

[10] Gaetan Lafortune GBaA, Durand OHD. Comparing activities and performance of the hospital sector in Europe: how many surgical procedures performed as inpatient and day cases? 2012.

[11] Kreindler SA. Policy strategies to reduce waits for elective care: a synthesis of international evidence. British Medical Bulletin. 2010; 95(1): 7-32. PMid: 20457662. https ://doi.org/10.1093/bmb/ $1 \mathrm{dq} 014$

[12] Demand and capacity - a comprehensive guide: NHS Improvement. Cited 202024 April]. Available from: https://improvement.nhs.uk/documents/2099/dema nd-capacity-comprehensive-guide.pdf 
[13] Mache S, Danzer G, Klapp BF, et al. Surgeons' work ability and performance in surgical care: relations between organisational predictors, work engagement and work ability. Langenbeck's Archives of Surgery. 2013; 398(2): 317-325. PMid: 23292431. https: //doi.org/10.1007/s00423-012-1044-3

[14] Tengland PA. The concept of work ability. Journal of Occupational Rehabilitation. 2011; 21(2): 275-285. PMid: 21052807. https://doi.org/10.1007/s10926-010-9269-x
[15] Demand and capacity models Core model user guidance England: NHS Improvemen; 2018 [cited 202024 April]. Available from: https://www. england.nhs.uk/wp-content/uploads/2018/ 11/demand-capacity-core-model-user-guidance.pdf

[16] AIHW: Elective surgery [Internet]. [cited 202024 April]. Available from: https://www.aihw.gov.au/reports-data/myho spitals/sectors/elective-surgery 